\title{
Neutrino induced weak pion production off the nucleon and coherent pion production in nuclei at low energies
}

\author{
J.E. Amaro*, E. Hernández ${ }^{\dagger}$, J. Nieves**, M. Valverde ${ }^{\ddagger}$ and M.J. Vicente-Vacas ${ }^{\S}$ \\ *Departamento de Física Atómica, Molecular y Nuclear, Universidad de Granada, E-18071 Granada, Spain \\ ${ }^{\top}$ Departamento de Física Fundamental e IUFFyM, Facultad de Ciencias, E-37008 Salamanca, Spain \\ ${ }^{* *}$ Instituto de Física Corpuscular (IFIC), Centro Mixto CSIC-Universidad de Valencia, Institutos de \\ Investigación de Paterna, Aptd. 22085, E-46071 Valencia, Spain \\ ${ }^{\sharp}$ Research Center for Nuclear Physics (RCNP), Osaka University, Ibaraki 567-0047, Japan \\ ${ }^{\S}$ Departamento de Física Teórica and IFIC, Centro Mixto CSIC-Universidad de Valencia, Institutos de \\ Investigación de Paterna, Aptd. 22085, E-46071 Valencia, Spain
}

\begin{abstract}
We present a microscopic model for neutrino induced one-pion production off the nucleon and its implementation for the purpose of calculating coherent pion production in nuclei. We further criticize the use of the Rein-Sehgal model for coherent pion production by low energy neutrinos. In particular, we show how the approximations in that model give rise to a much flatter differential cross section in the $\eta=E_{\pi}\left(1-\cos \theta_{\pi}\right)$ variable. We discuss the limitations intrinsic to any approach based on the partial conservation of the axial current hypothesis and the inability of such models to properly determine the angular distribution of the outgoing pion with respect to the direction of the incoming neutrino. We show the effects of those limitation for the case of the $\frac{d \sigma}{d \eta}$ differential cross section.
\end{abstract}

Keywords: Neutrino reactions, coherent pion production

PACS: $25.30 . \mathrm{Pt}, 12.15 .-\mathrm{y}, 13.15 .+\mathrm{g}$

\section{INTRODUCTION}

Neutrino induced one-pion production off nucleons and nuclei in the intermediate energy region is a source of relevant data on hadronic structure. Pions are mainly produced through resonance excitation and these reactions can be used to extract information on nucleon-to-resonance axial transition form factors. Besides, a proper understanding of these processes is very important in the analysis of neutrino oscillation experiments since pion production could be a source of background in those experiments [1,2].

In reactions on nuclei, pions can be produced incoherently or coherently. In the latter case the nucleus remains in its ground state. Coherent reactions are controlled by the nucleus form factor and are more forward peaked than incoherent ones. Experimental analyses of the coherent reaction rely on the Rein-Sehgal (RS) model [3] which assumes that coherent pion production is dominated by the divergence of the axial current and can thus be related to the pionnucleus coherent scattering through the partial conservation of the axial current (PCAC) hypothesis. For instance for coherent $\pi^{0}$ production the RS model approximates the coherent cross section for both neutrino and antineutrino induced processes by

$$
\frac{d \sigma}{d x d y d t}=\frac{G^{2} M E}{\pi^{2}} f_{\pi}^{2}(1-y) \frac{1}{\left(1-q^{2} / 1 \mathrm{GeV}^{2}\right)^{2}}\left(\left.\left|F_{\mathscr{A}}(t)\right|^{2} F_{\mathrm{abs}} \frac{d \sigma\left(\pi^{0} N \rightarrow \pi^{0} N\right)}{d t}\right|_{E_{\pi}=q^{0}, t=0}\right),
$$

with $G$ the Fermi constant, $M$ the nucleon mass, $E$ the incident neutrino energy and $f_{\pi}$ the pion decay constant. Besides $q$ is the lepton four-momentum transfer, $x=-q^{2} / 2 M q^{0}, y=q^{0} / E$, and $t=\left(q-k_{\pi}\right)^{2}$ with $k_{\pi}$ the pion four-momentum. $t$ equals $-\left(\vec{q}-\vec{k}_{\pi}\right)^{2}$ if, as usually assumed, the nucleus recoil is neglected $\left(q^{0}=k_{\pi}^{0} \equiv E_{\pi}\right) . F_{\mathscr{A}}(t)$ is the the nuclear form factor calculated as $F_{\mathscr{A}}(t)=\int d^{3} \vec{r} e^{\mathrm{i}\left(\vec{q}-\vec{k}_{\pi}\right) \cdot \vec{r}}\left\{\rho_{p}(\vec{r})+\rho_{n}(\vec{r})\right\}$ with $\rho_{p(n)}$ the nuclear proton (neutron) density, normalized to the number of protons (neutrons). $F_{\text {abs }}$ is a $t$-independent attenuation factor that takes into account the distortion of the final pion. Finally $\left.\frac{d \sigma\left(\pi^{0} N \rightarrow \pi^{0} N\right)}{d|t|}\right|_{q^{0}=E_{\pi}, t=0}$ is the differential pion-nucleon cross section evaluated at $t=0$. The $t=0$ approximation in the pion-nucleon cross section is not needed and it can be justified only if the nuclear form factor is sufficiently forward peaked. The larger the pion energy and the heavier the nucleus, the better this approximation becomes. In the original paper [3], the model was applied to medium size nucleus, aluminum, and 
neutrino energies above $2 \mathrm{GeV}$, for which the relevant pion energies are quite high. However, as we pointed out in [4], for neutrino energies below $1 \mathrm{GeV}$ and lighter nuclei, like carbon or oxygen, the nuclear form factor is not enough forward peaked to render the finite $t$-dependence of the pion-nucleon cross section negligible. The distortion factor $F_{\text {abs }}$ is also an oversimplification since in any realistic scattering model this factor should depend on $t$. The recent work of Berger and Sehgal [5] already corrects some of the problems in the RS model.

In Ref. [6] we discuss in detail the approximations inherent to any PCAC based model and, in particular, the approximations in the RS model. As we show below, the neglect of non-PCAC terms and the implicit assumption (only correct for $q^{2}=0$ ) by PCAC based models that $\frac{d \sigma}{d x d y d t d \phi_{k \pi q}}=\frac{1}{2 \pi} \frac{d \sigma}{d x d y d t}$, where $\phi_{k_{\pi} q}$ is the azimuthal pion angle in a plane perpendicular to $\vec{q}$, affects the determination of the differential cross sections with respect to the angle made by the pion and the incident neutrino, leading to flatter distributions in that variable. In the particular case of the RS model the $t=0$ approximation enhances this unwanted effect. This could have implications for the recent determination by the MiniBooNE Collaboration of the neutral current (NC) coherent $\pi^{0}$ production rate [7].

Our approach to the problem, as others in the literature, does not rely on PCAC. We use a microscopic model in which coherent pions are mainly produced by virtual $\Delta$-hole excitations in the nucleus, as well as additional processes that are required by chiral symmetry. The model includes the modifications of the $\Delta$ propagator in the nucleus by means of a sophisticated evaluation of the $\Delta$ self-energy in the medium, and it treats the final pion distortion in a realistic way by solving the Klein-Gordon $(\mathrm{KG})$ equation for a pion-nucleus optical potential.

\section{MICROSCOPIC MODEL AT THE NUCLEON LEVEL}

The model that we use to describe the elementary production process at the nucleon level is the one derived in Ref. [8]. In Fig. 1 we give the different contributions for the charged current (CC) $W^{+} N \rightarrow N^{\prime} \pi$ case (details for the antineutrino induced process and the $\mathrm{NC}$ case can be found in [8]). In addition to the dominant $\Delta$ pole $(\Delta P)$ mechanism (weak excitation of the $\Delta(1232)$ resonance and its subsequent decay into $N \pi$ ), the model also includes the crossed $\Delta$ pole term $(C \Delta P)$ and other background terms that are required by chiral symmetry: Direct and crossed nucleon (second row) pole terms ( $N P, C N P$ ), contact $(C T)$ and pion pole $(P P)$ contribution (third row) and finally the pion-in-flight $(P F)$ term. In Ref. [8], we found that background terms produced significant effects in all channels. The least known
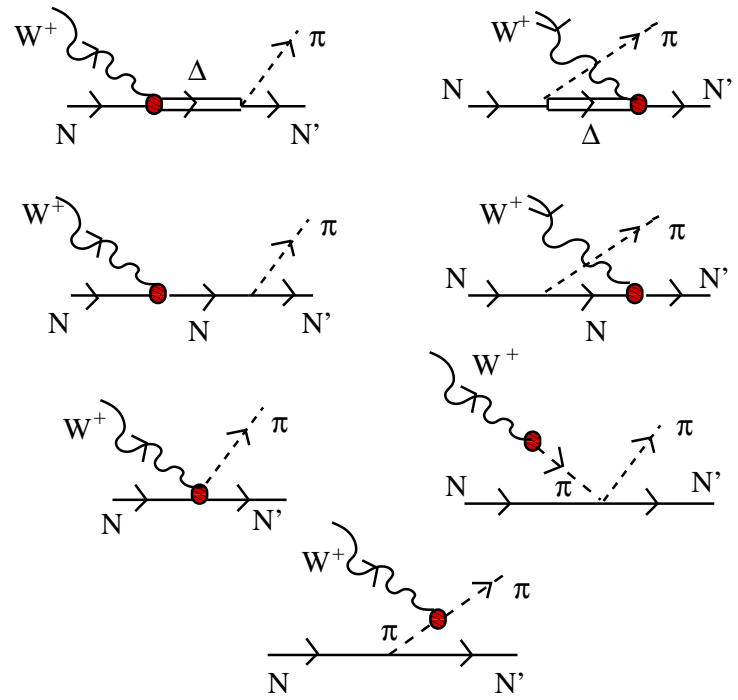

FIGURE 1. Model of Ref. [8] for the $W^{+} N \rightarrow N^{\prime} \pi$ reaction. The circle in the diagrams stands for the weak transition vertex.

ingredients of the model are the axial nucleon-to- $\Delta$ transition form factors, of which $C_{5}^{A}$ gives the largest contribution (See Eq.(1) of Ref. [9] for a form factor decomposition of the $N \Delta$ weak current). Besides, within the Adler model that we use [10], $C_{5}^{A}$ determines all other axial form factors. This strongly suggested the readjustment of that form factor to the experimental data, which we did by fitting the flux-averaged $v_{\mu} p \rightarrow \mu^{-} p \pi^{+}$ANL $[11,12] q^{2}$-differential cross section for pion-nucleon invariant masses $W<1.4 \mathrm{GeV}$, for which the model should be appropriate. Assuming the 
shape [13]

$$
C_{5}^{A}\left(q^{2}\right)=\frac{C_{5}^{A}(0)}{\left(1-q^{2} / M_{A \Delta}^{2}\right)^{2}} \times \frac{1}{1-\frac{q^{2}}{3 M_{A \Delta}^{2}}}
$$

we found $C_{5}^{A}(0)=0.867 \pm 0.075$ and $M_{A \Delta}=0.985 \pm 0.082 \mathrm{GeV}$. Our full model, thus obtained, lead to an overall better description of the data for different $\mathrm{CC}$ and $\mathrm{NC}$, neutrino- and antineutrino-induced, one-pion production reactions off the nucleon [8]. The $C_{5}^{A}(0)$ value is significantly smaller than the traditionally used value of about 1.2 deduced from the off-diagonal Goldberger-Treiman relation. This reduction of $C_{5}^{A}(0)$ is consistent with recent results in lattice QCD [14], quark model [15] and phenomenological studies [16].

\section{COHERENT PRODUCTION IN NUCLEI: MODEL AND RESULTS}

Now the process consists of a weak pion production followed by the strong distortion of the pion in its way out of the nucleus. In the coherent production the nucleus is left in its ground state and the hadronic amplitude is written as a sum over the amplitudes for each nucleon. Neglecting for the moment nonlocalities and pion distortion one can write for the hadronic amplitude

$$
\mathscr{A}_{\pi}^{\mu}\left(q, k_{\pi}\right)=\int d^{3} \vec{r} e^{\mathrm{i}\left(\vec{q}-\vec{k}_{\pi}\right) \cdot \vec{r}}\left\{\rho_{p}(\vec{r})\left[\mathscr{J}_{p \pi}^{\mu}\left(q, k_{\pi}\right)\right]+\rho_{n}(\vec{r})\left[\mathscr{J}_{n \pi}^{\mu}\left(q, k_{\pi}\right)\right]\right\}
$$

where the approximation $q^{0}=k_{\pi}^{0}$ (neglect of nucleus recoil) has been used. One can see from Eq.(3) that the coherent pion production process is sensitive to the Fourier transform of the nuclear density for momentum $\vec{q}-\vec{k}_{\pi} \cdot \mathscr{J}_{N \pi}^{\mu}\left(q, k_{\pi}\right)$ in Eq.(3), stands for the nucleon spin averaged $W N \rightarrow N \pi$ or $Z^{0} N \rightarrow N \pi$ weak transition amplitude

$$
\mathscr{J}_{N \pi}^{\mu}\left(q, k_{\pi}\right)=\frac{1}{2} \sum_{r} \bar{u}_{r}\left(\vec{p}^{\prime}\right) \Gamma_{i ; N \pi}^{\mu} u_{r}(\vec{p}) \frac{M}{p^{0}}, \quad i=\Delta P, C \Delta P, N P, C N P, C T, P P, P F
$$

where the $u$ 's are Dirac spinors for the nucleons, normalized such that $\bar{u} u=2 M$, and the four-vector matrices $\Gamma_{i ; N \pi}^{\mu}$ can be read from the explicit expressions of the pion production amplitudes given in Ref. [8]. $\vec{p}$ and $\vec{p}^{\prime}=\vec{p}+\vec{q}-\vec{k}_{\pi}$ are the initial and final three momenta of the nucleon. Those momenta are not well defined and we approximate the four-momentum of the initial nucleon by $\left.p^{\mu}=\sqrt{M^{2}+\frac{1}{4}\left(\vec{k}_{\pi}-\vec{q}\right)^{2}}, \frac{\vec{k}_{\pi}-\vec{q}}{2}\right)$. Hence we assume that the initial nucleon momentum is $\left(\vec{k}_{\pi}-\vec{q}\right) / 2$ and the final one is $-\left(\vec{k}_{\pi}-\vec{q}\right) / 2$, with both nucleons being on-shell. The momentum transfer is equally shared between the initial and final nucleon momenta. Setting $\vec{p}=-\vec{p}^{\prime}=\left(\vec{k}_{\pi}-\vec{q}\right) / 2$ eliminates some nonlocal contributions, and it greatly simplifies the sum over all nucleons, which can be cast in terms of the neutron and proton densities. Furthermore, the sum over spins in Eq. (4) can be also easily performed for $\vec{p}=-\vec{p}^{\prime}$ since $u_{r}\left(\vec{p}^{\prime}=-\vec{p}\right)=\gamma^{0} u_{r}(\vec{p})$, so that

$$
\frac{1}{2} \sum_{r} \bar{u}_{r}\left(\vec{p}^{\prime}=-\vec{p}\right) \Gamma_{i ; N \pi}^{\mu} u_{r}(\vec{p})=\frac{1}{2} \operatorname{Tr}\left((\not p+M) \gamma^{0} \Gamma_{i ; N \pi}^{\mu}\right), \quad i=\Delta P, C \Delta P, N P \ldots
$$

Given the importance of the $\Delta$-pole contribution and since the $\Delta$ properties are strongly modified inside the nuclear medium we consider some additional nuclear corrections to this contribution to include the effect of the self-energy of the $\Delta$ in the medium $\Sigma_{\Delta}(\rho(\vec{r}))$. We follow the same approach as in Ref. [17], which is based on the findings of Refs. $[18,19,20]$. Thus in the $\Delta$-propagator, we make the substitutions $M_{\Delta} \rightarrow M_{\Delta}+\operatorname{Re} \Sigma_{\Delta}$ and $\Gamma_{\Delta} / 2 \rightarrow \Gamma_{\Delta}^{\text {Pauli }} / 2-$ $\operatorname{Im} \Sigma_{\Delta}$ and take $\Sigma_{\Delta}(\rho(\vec{r}))$ and $\Gamma_{\Delta}^{\text {Pauli }} / 2$ as explained in Sect. II-B of Ref. [17]. Once these corrections are included $\mathscr{J}_{N \pi}^{\mu}$ depends on $\vec{r}$ as well and the nuclear form factor can no longer be factorized out as in the RS model.

So far the formalism has used the bound wave functions of the nucleons in the nucleus, which appear via the proton and neutron densities, and has considered only a plane wave for the pion. Pion distortion effects are important, specially for $\left|\vec{k}_{\pi}\right|<0.5 \mathrm{GeV}$, and are considered by replacing in Eq. (3)

$$
\begin{array}{r}
e^{-\mathrm{i} \vec{k}_{\pi} \cdot \vec{r}} \rightarrow \widetilde{\varphi}_{\pi}^{*}\left(\vec{r} ; \vec{k}_{\pi}\right) \\
\vec{k}_{\pi} e^{-\mathrm{i} \vec{k}_{\pi} \cdot \vec{r}} \rightarrow \mathrm{i} \vec{\nabla} \widetilde{\varphi}_{\pi}^{*}\left(\vec{r} ; \vec{k}_{\pi}\right)
\end{array}
$$




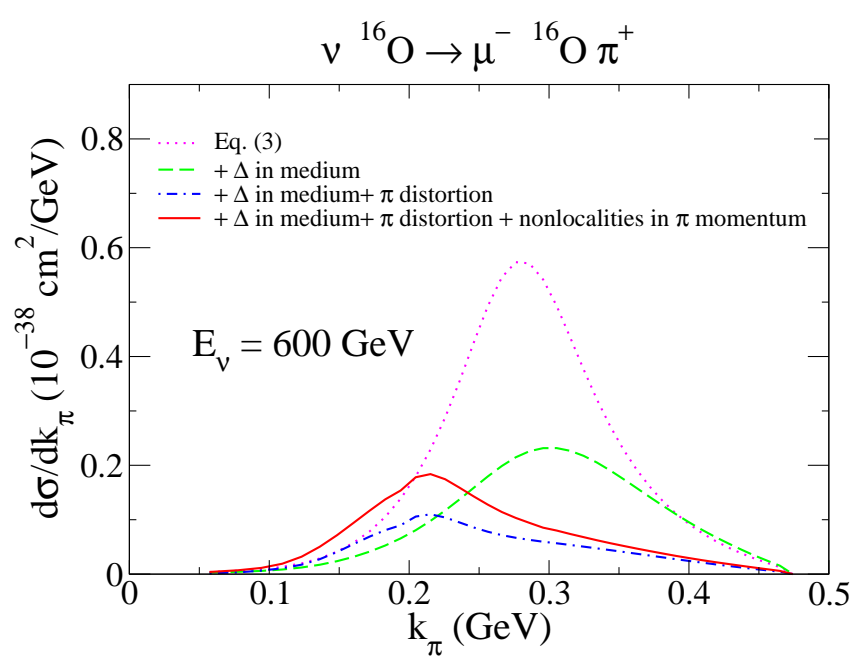

FIGURE 2. Effects of $\Delta$ in medium, $\pi$ distortion and nonlocalities in $\pi$ momentum on the $\frac{d \sigma}{d k_{\pi}}$ differential cross section evaluated for the $v^{16} O \rightarrow \mu^{-16} O \pi^{+}$reaction at $E_{v}=0.6 \mathrm{GeV}$.

The pion wave function $\widetilde{\varphi}_{\pi}^{*}\left(\vec{r} ; \vec{k}_{\pi}\right)$ corresponds to an incoming solution of the KG equation,

$$
\left[-\vec{\nabla}^{2}+m_{\pi}^{2}+2 E_{\pi} V_{\mathrm{opt}}(\vec{r})\right] \widetilde{\varphi}_{\pi}^{*}\left(\vec{r} ; \vec{k}_{\pi}\right)=E_{\pi}^{2} \widetilde{\varphi}_{\pi}^{*}\left(\vec{r} ; \vec{k}_{\pi}\right)
$$

with $V_{\text {opt }}(\vec{r})$ the optical potential which describes the $\pi$-nucleus interaction. This potential has been developed microscopically and it is explained in detail in Refs. $[19,20]$. This theoretical potential reproduces fairly well the data of pionic atoms (binding energies and strong absorption widths) [19] and low energy $\pi$-nucleus scattering [20]. At low pion energies, it is an improvement over the one used in [17, 21], that was based on $\Delta$ dominance of the $\pi N$ interaction. The replacement in Eq. (7), that takes into account the fact that the pion three momentum is only well defined asymptotically when the pion-nucleus potential vanishes, induces some non-localities in the amplitudes. To treat these non-localities we have adopted the following scheme:

- In the $\Delta P, C \Delta P, N P, C N P$ terms, we note that there exist either a $N N \pi$ or a $N \Delta \pi$ vertex (see Eq. (51) of Ref. [8]), which induces a factor $k_{\pi}^{\alpha}$ in the amplitudes. Indeed, for those terms we could re-write

$$
\mathscr{J}_{i ; N \pi}^{\mu}\left(\vec{r} ; q, k_{\pi}\right)=\left(k_{\pi}\right)_{\alpha} \hat{\mathscr{J}}_{i ; N \pi}^{\mu \alpha}\left(\vec{r} ; q, k_{\pi}\right), \quad i=\Delta P, C \Delta P, N P, C N P .
$$

We do not consider any non-locality in the tensor $\hat{\mathcal{J}}_{i ; N \pi}^{\mu \alpha}$, and we use the prescription of Eqs. (6) and (7) to account for $\vec{k}_{\pi}$ in the contraction between $k_{\pi}^{\alpha}$ and $\hat{J}_{i ; N \pi}^{\mu \alpha}$ in Eq. (9). For $k_{\pi}^{0}$ we shall use the asymptotic pion energy. This approach to treat the non-localities is equivalent to that assumed in Refs. [17, 21].

- We do not consider any non-locality for the $C T$ and $P P$ contributions.

The effects of the modifications of the $\Delta$ properties in the medium, pion distortion and nonlocalities in the pion momentum are shown in Fig. 2. As can be seen all corrections are very relevant. Very recently, it has been claimed that nonlocalities in the nucleon momentum could be as important, giving rise to a large reduction of the coherent cross section [22]. The calculation in Ref. [22] has been done without $\Delta$ in medium corrections or pion distortion and further studies are needed to clarify this point.

As mentioned before, background terms gave an important contribution at the nucleon level [8] but they turned out to be irrelevant for coherent production in symmetric nuclei [4], and thus the reduction we found in $C_{5}^{A}(0)$ amounts to an important decrease in the coherent cross section. In Fig. 4 we give cross sections for $\mathrm{CC}$ and $\mathrm{NC}$ coherent pion production on carbon and oxygen. Other observables are discussed in Ref. [4].

Let us here concentrate on the $\frac{d \sigma}{d \eta}$ differential cross section with $\eta=E_{\pi}\left(1-\cos \theta_{\pi}\right)$ the variable introduced by the MiniBooNE Collaboration in its analysis of NC coherent $\pi^{0}$ production [7]. In Fig. 4 we show results for NC coherent $\pi^{0}$ production on carbon at $E_{v}=1 \mathrm{GeV}$. In the left panel the results have been evaluated without distortion or any 

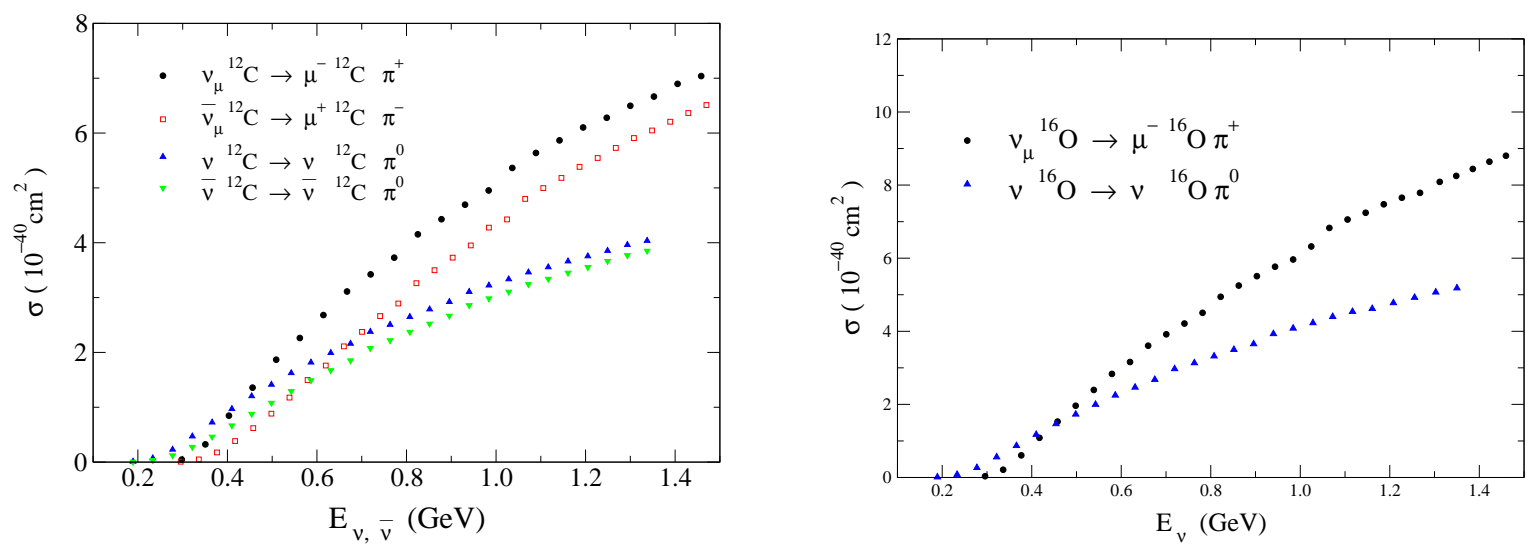

FIGURE 3. Muon neutrino/antineutrino CC and NC coherent pion production off nuclei from carbon (left) and oxygen (right) targets as a function of the neutrino/antineutrino energy.
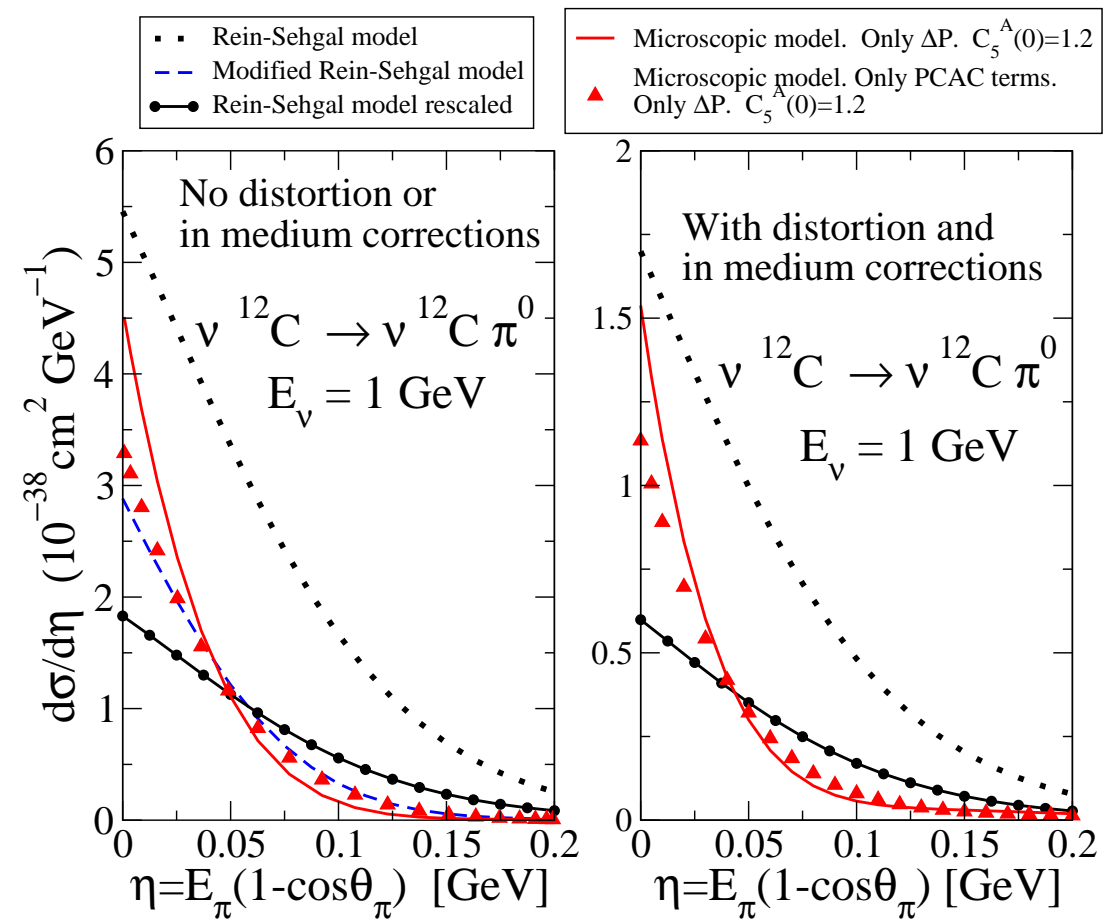

FIGURE 4. Comparison of different predictions for the $\frac{d \sigma}{d \eta}$ differential cross section for NC coherent $\pi^{0}$ production on carbon at $E_{v}=1 \mathrm{GeV}$. See text for details.

in medium correction. We compare the results obtained with the RS model, the one used by experimentalists in their analyses, with the results obtained in the microscopic model of Refs.[8, 4]. In the latter case and for simplicity, we have only taken the dominant $\Delta P$ term and, in order to fix the normalization, we use $C_{5}^{A}(0)=1.2$ which is the value implicit in any PCAC-based model. Besides we show, with triangles, results of the microscopic model eliminating all non-PCAC contributions. Comparison of the latter with the RS model shows the effect of the $t=0$ approximation used in that model. Cross sections are larger in the RS model and what is more important this model produces a much flatter distribution (see line "Rein-Sehgal rescaled"). As described in Ref. [6], one can easily eliminate the $t=0$ approximation in the RS model by using the full non spin-flip part of the pion nucleon elastic cross section. The results for this case are shown under "Modified Rein-Sehgal" with a dashed line. We see the agreement with the only-PCACterms microscopic model is very good in this case. Another important piece of information can be extracted from 
the comparison between the full microscopic calculation and the only-PCAC-terms one. We see the latter produces a flatter distribution. This is mainly due to the $\frac{d \sigma}{d x d y d t d \phi_{k \pi q}}=\frac{1}{2 \pi} \frac{d \sigma}{d x d y d t}$ approximation inherent to any PCAC-based model. In the right panel we show results that include distortion and in medium corrections. Again we see the RS model produces larger and much flatter distributions than the microscopic calculation due to the $t=0$ approximation and the inadequacy of the final pion distortion used. Besides the effect of the $\frac{d \sigma}{d x d y d t d \phi_{k \pi q}}=\frac{1}{2 \pi} \frac{d \sigma}{d x d y d t}$ approximation can also be seen by comparing the two microscopic calculations.

\section{CONCLUSIONS}

To conclude we would like to stress the following: First, background terms are relevant at the nucleon level and should be included in any microscopic calculation. Even though they play a minor role in coherent production, their inclusion affects the determination of the $C_{5}^{A}(0)$ value which is a crucial quantity for coherent production as it fixes the strength of the dominant $\Delta P$ contribution. Second, the Rein-Sehgal model was cleverly devised for its use at high neutrino energies and medium/large nuclei but we think we have shown it is inadequate for low neutrino energies and small nuclei. The use of the RS model in experimental analyses could affect the determination of the rate of coherent production. We believe this is the case for the recent determination of $\mathrm{NC} \pi^{0}$ coherent production by the MiniBooNE Collaboration [7]. Last, some of the problems of the RS model are shared by all PCAC-based models: the neglect of non-PCAC terms and the implicit assumption $\frac{d \sigma}{d x d y d t d \phi_{k \pi q}}=\frac{1}{2 \pi} \frac{d \sigma}{d x d y d t}$ makes those models unable to properly determine differential cross sections with respect to any variable that depends on the angle made by the pion and the incident neutrino. We have shown that for the case of the $\frac{d \sigma}{d \eta}$ differential cross section.

\section{ACKNOWLEDGMENTS}

This research was supported by DGI and FEDER funds, under contracts FIS2008-01143/FIS, FIS2006-03438, FPA2007-65748, and the Spanish Consolider-Ingenio 2010 Programme CPAN (CSD2007-00042), by Junta de Castilla y León under contracts SA016A07 and GR12, and it is part of the European Community-Research Infrastructure Integrating Activity "Study of Strongly Interacting Matter" ( HadronPhysics2, Grant Agreement n. 227431) under the Seventh Framework Programme of EU. M.V. wishes to acknowledge a postdoctoral fellowship form the Japan Society for the Promotion of Science.

\section{REFERENCES}

1. A. A. Aguilar-Arevalo et al. [The MiniBooNE Collaboration], Phys. Rev. Lett. 98, 231801 (2007).

2. K. Hiraide [SciBooNE Collaboration], Nucl. Phys. Proc. Suppl. 159, 85 (2006).

3. $\quad$ D. Rein and L. M. Sehgal, Nucl. Phys. B 8223, 29 (1983).

4. J. E. Amaro, E. Hernández, J. Nieves and M. Valverde, Phys. Rev. D 79, 013002 (2009).

5. $\quad$ C. Berger and L. M. Sehgal, Phys. Rev. D 79, 053003 (2009).

6. E. Hernandez, J. Nieves and M. J. Vicente-Vacas, Phys. Rev. D 80, 013003 (2009).

7. A. A. Aguilar-Arevalo et al. [MiniBooNE Collaboration], Phys. Lett. B 664, 41 (2008).

8. $\quad$ E. Hernández, J. Nieves and M. Valverde, Phys. Rev. D 76, 033005 (2007).

9. P. A. Schreiner and F. Von Hippel, Phys. Rev. Lett. 30, 339 (1973).

10. $\quad$ S.L. Adler, Ann. Phys. 50 (1968) 189.

11. S. J. Barish et al., Phys. Rev. D 19 (1979) 2521.

12. G. M. Radecky et al., Phys. Rev. D 25 (1982) 1161. [Erratum-ibid. D 26 (1982) 3297].

13. E.A. Paschos, J.-Y. Yu and M. Sakuda, Phys. Rev. D69 (2004) 014013.

14. C. Alexandrou, T. Leontiou, J. W. Negele and A. Tsapalis, Phys. Rev. Lett. 98, 052003 (2007).

15. D. Barquilla-Cano, A. J. Buchmann and E. Hernandez, Phys. Rev. C 75, 065203 (2007). [Erratum-ibid. C 77, 019903 (2008)].

16. K. M. Graczyk and J. T. Sobczyk, Phys. Rev. D 77, 053001 (2008).

17. L. Alvarez-Ruso, L. S. Geng, S. Hirenzaki and M. J. Vicente Vacas, Phys. Rev. C 75, 055501 (2007).

18. E. Oset and L. L. Salcedo, Nucl. Phys. A 468, 631 (1987).

19. J. Nieves, E. Oset and C. Garcia-Recio, Nucl. Phys. A 554, 509 (1993).

20. J. Nieves, E. Oset and C. Garcia-Recio, Nucl. Phys. A 554, 554 (1993).

21. L. Alvarez-Ruso, L. S. Geng and M. J. Vicente Vacas, Phys. Rev. C 76, 068501 (2007).

22. T. Leitner, U. Mosel and S. Winkelmann, Phys. Rev. C 79 (2009) 057601. 